provide all levels of oncologic care. The aim of this study was to assess the need for palliative care in hospitalised cancer patients in Mato Grosso do Sul, one of the Brazilian states that has no public hospital offering this type of care.

Methods A descriptive study of all hospitalisations due to "complications of clinical oncology (ICPO)" in hospitals of the Unified Health System (SUS) in the state of Mato Grosso do Sul, Brazil, was conducted from January 2008 to August 2010. Data were obtained from the Hospital Information System of the Unified Health System (SIH-SUS).

Results There were 5165 hospitalisations for ICPO with cancer as the underlying cause in $94.7 \%$. Average length of stay was 6.2 days and 1189 patients $(23.0 \%)$ died. Individuals aged 65 years or older accounted for $44.3 \%$ of these deaths and for every 3.5 hospital admissions in this age group, one ended in death. Many patients died without the appropriate care.

Conclusion The ageing of the population and the increased incidence of cancer brought new challenges to the Brazilian Unified Health System, including the need for palliative care. This need is not always met for instance as in Mato Grosso do Sul. In this sense, deaths of patients hospitalised for ICPO can be thought of as indicators of advanced disease and they could be useful to national and state policies for oncologic palliative care.

\section{P1-233 ASSESSING THE EFFECT OF INDIVIDUAL AND AREA LEVEL SOCIODEMOGRAPHIC FACTORS ON REGIONAL DIFFERENCES IN MORTALITY AMENABLE TO HEALTHCARE}

doi:10.1136/jech.2011.142976e.26

\begin{abstract}
${ }^{1,2} \mathrm{~A}$ McCallum, ${ }^{3} \mathrm{M}$ Arfmann, ${ }^{4} \mathrm{~A}$ Leyland, ${ }^{3} \mathrm{~S}$ Karvonen, ${ }^{3} \mathrm{~K}$ Manderbacka, ${ }^{3}$ K Keskimaki. ${ }^{1}$ NHS Lothian, Edinburgh, UK; ${ }^{2}$ University of Edinburgh, Edinburgh, UK; ${ }^{3}$ National Institute for Health and Welfare, Helsinki, Finland; ${ }^{4}$ MRC Social and Public Health Sciences Unit, Glasgow, UK
\end{abstract}

Introduction Mortality from many causes varies geographically. We examine trends in regional differences in amenable mortality in Finland and explore the role of socioeconomic factors.

Methods We analysed deaths amenable to primary and specialist healthcare in the Finnish population aged $25-74$ in 1992-2003. Sociodemographic variables were individually linked from annual employment statistics. Three area-level scores were created using factor analysis of municipal level register data; these represented standard of living, deprivation and (poor) social cohesion. Multilevel Poisson regression models were applied adjusting for sex and age, other individual-level variables and area-level factor scores.

Results Mortality from conditions amenable to primary care intervention was more common among men in 1992-1995 but differences disappeared over time. An inverse income gradient increased over time. The unemployed and those outside the labour force had higher mortality. Poor social cohesion of the area was also associated with mortality. Men had more than double the risk of dying from conditions related to specialist care compared to women. An inverse and increasing gradient was seen for income. Employment status was again associated with higher mortality. Poor social cohesion of the area increased risk as did deprivation in 1996-2003. There was little variation between areas for conditions amenable to primary healthcare, but substantial variation for mortality amenable to specialist healthcare.

Conclusion Mortality amenable to primary care is strongly patterned by individual socio-economic circumstances. Conditions amenable to specialist care also show strong social gradients but large area variances suggest that the organisation and delivery of specialist services may also influence mortality.
P1-234 PRENATAL SCREENING FOR SUBOPTIMAL MENTAL HEALTH IN THE POSTPARTUM PERIOD

doi:10.1136/jech.2011.142976e.27

S McDonald, ${ }^{*} \mathrm{~J}$ Wall, K Forbes, S Tough. University of Calgary, Calgary, Alberta, Canada

Postpartum depression (PPD) is the most common complication of pregnancy in developed countries, affecting $10 \%-15 \%$ of all new mothers. There's been a shift in thinking less in terms of PPD per se to poor psychosocial and transitioning outcomes after giving birth. The objective of this study was to develop a screening tool that identifies women at risk of distress in the postpartum period using information collected prenatally. We used data collected for the All Our Babies Study, a prospective cohort study of pregnant women living in Alberta, Canada $(\mathrm{N}=1578)$ that collects a diverse array of information at three time points during the perinatal period. We developed the tool using $2 / 3$ of the sample and performed internal validation on the remaining $1 / 3$ using a regression coefficient-based scoring method. The best fit model included known risk factors for PPD and suboptimal psychosocial health: depression and stress in late pregnancy, history of abuse, and poor relationship quality with partner. The area under the ROC curve was 0.76 , with acceptable sensitivity and specificity for a cut-off score of 2 (range $0-7$ ). Comparison of the tool with a widely used PPD screening inventory showed that our tool had better performance indicators. Further validation of our tool for psychosocial distress was seen in its utility for identifying symptoms of anxiety, in addition to depression, at 4 months. There is an opportunity for early detection of risk to inform the development of interventions to prevent difficulties and promote optimal well-being for mothers and their families.

\section{P1-235 DOES SOCIAL SUPPORT BUFFER ANXIETY ASSOCIATED WITH RETURNING TO WORK OR SCHOOL FOR NEW MOTHERS IN THE FIRST YEAR AFTER GIVING BIRTH?}

doi:10.1136/jech.2011.142976e.28

S McDonald, ${ }^{*}$ H Kehler, S Tough. University of Calgary, Calgary, Alberta, Canada

In Canada, maternity leave can last up to 1 year after giving birth. For some women, there may be associated anxiety with the prospect of having to return to work or school. We investigated the extent to which employment intentions during early postpartum were associated with symptoms of anxiety and whether this association was moderated by perceptions of social support. We used data from the All Our Babies Study, a community based prospective cohort study of pregnant women in Calgary, Alberta, Canada $(n=1578)$. Women completed confidential questionnaires that collected information about their pregnancy, lifestyle, life events, social support, stress, and mental health at three time points: early pregnancy, late pregnancy, and 4 months postpartum. We asked women detailed questions about their intentions of returning to work or school at the 4 month data collection. Women who noted that they would be returning to work or school within 12 months had significantly higher anxiety symptomatology, controlling for SES and baseline anxiety, than women who were not returning to work or school. We classified women into four mutually exclusive categories according to timing of return to work/school and perceived social support in order to examine the buffering hypothesis of social support. The group of women that manifested the most anxiety comprised those with intentions of returning when their infants were $<12$ months of age AND low perceived social support. These findings have implications for developing targeted strategies to support working mothers. 